\title{
RainWatch Project: Location-Awared Realtime Detection and Notification of Rain on Internet-Based Sensor Network
}

\author{
Kaveevivitchai Sathita \\ Hideya Ochiai \\ Hiroshi Esaki \\ The University of Tokyo, 7-3-1 Hongo, Bunkyo-ku, Tokyo, 113-8656, Japan \\ wan@hongo.wide.ad.jp jo2lxq@hongo.wide.ad.jp hiroshi@wide.ad.jp
}

\begin{abstract}
This paper introduces RainWatch project which enable rain-sensitive users to monitor real time rain information in area of users' interest. Real-time detection and notification of rain situation will allow users to take appropriate preparation (e.g., evacuation from the area in case of disaster prevention), which can prevent loss of lives and damage on our properties. The RainWatch system stands on top of Live E! data platform, which is a wide-area sensor application testbed operated by multiple-organizations distributedly with about 100 Internet weather stations. The RainWatch system monitors the rain state around a certain geographical point and makes notifications or warnings (e.g., the rain is approaching) in real-time to the users that have interest in the point. Strong needs for this application will influence the design of the sensor networking infrastructure regard to location-awareness and real-time-ness.
\end{abstract}

\section{INTRODUCTION}

Internet weather stations have enabled realtime collection of environmental data over wide geographical areas. The collected data should be practically managed in a distributed manner because of operational reasons (e.g., multiple-organizations) and of the vast data amount. On the other end, there will be many types of data retrieval requests generated by user applications. To design the system that provides sufficient performance for those requests is one of the major issues in this research area.

This paper describes RainWatch Project as one of the applications that provide information in term of users/sensors' location-aware. This project is aimed to provide useful-information for rain-sensitive end users via e-mail or by other means; e.g., (1) the rain has been observed within $6 \mathrm{~km}$ around this point, or (2) the rain is approaching toward this point. This RainWatch service extracts a small set (specified by geographical-area) of raw data from the massive dataset, analyzes and summarizes it into user-friendly format. In other word, RainWatch is the application, which aggregate interested raw data from the location-aware dataset. This application provides the idea in designing Internet-based sensor networks applications.

RainWatch project is one of the approaches to tackle on recent natural disasters, which causes lost of lives and destructions; e.g., torrential rain, flash flooding and/or heat island effects in the metropolitan. By observer (end-users) able to receive prior notice about the rain that will occur shortly, they can take appropriate actions in order to minimize those possible effects.

We recognize that there are existing methods which provide rain forecast based on Surface Weather Observation, Weather satellites, IR-image, Meteorological radar, together with prediction models [1]. However, this RainWatch project takes advantage of real-time-ness of the system for watching the current rain situation around Point of Interest (POI) and provides the report or notification to the end-users. So they can further take actions in preparation for the coming rain. This output can be used in integral with other methods to provide more accurate notifications or warnings for the endusers.

We deploy the RainWatch system over Live E! sensor networking platform [2]. Sensor readings from about 100 Internet weather stations are distributedly managed on the Live E! data platform, and users can retrieve those data for their own activities. By applying the RainWatch system to the real sensor testbed, it is expected that we will get the beneficial examples as follow:

- Workers in an outdoor construction site must care about the rain status of their location for their own safety. The workers can halt their work and move out from the field before the rain hits the site.

- By previously knowing about the coming rain, stores can pack up products that set outdoor to prevent them from damage

- Stores can also enhance some sales such as sale of umbrella by preparing it to be just in grasp for the customers

- Disaster prevention in case of flash flooding can be achieved by prior warning

\section{LIVE E! PROJECT}

Live E! Project is an open research consortium established by means of collaboration among industry and many academic institutions. This project provides platform for sharing digital environmental data, currently focusing on weather data namely; temperature, humidity, pressure, rainfall, wind speed, and wind direction. These data are collected by sensor nodes embedded with Internet 
connectivity which allows the data to be available over the network in real-time. Sensors are installed and operated individually by interested stakeholders which allow them to benefits from the collected data however the data is also opened for the public.

Live E! has adopted three-tiered architecture for managing the system (Figure 1). The system is composed of (1) sensor layer, (2) data management layer and (3) application layer. The sensors, at the bottom layer, observe the environmental data and transmit the result to the data management platform. The data management platform, at the middle layer, is operated by multiple organizations collaboratively over the Internet in a distributed manner. The applications, at the top layer, fetch interested data from the data platform. There are three main areas of application, for education material, for public services, e.g., disaster reduction, and for business usages [3].

The Live E! Data platform works as a testbed for such applications. In February 2009, Live E! Project has 106 weather stations across 13 countries, and the platform is operated by 9 organizations. Kurashiki-city, Okayama, Japan has high density of weather stations where 26 sensors are deployed in $25 \mathrm{~km} \times 25 \mathrm{~km}$. The RainWatch system uses the data of this area as its testbed, due to the number of its sensors per square unit.

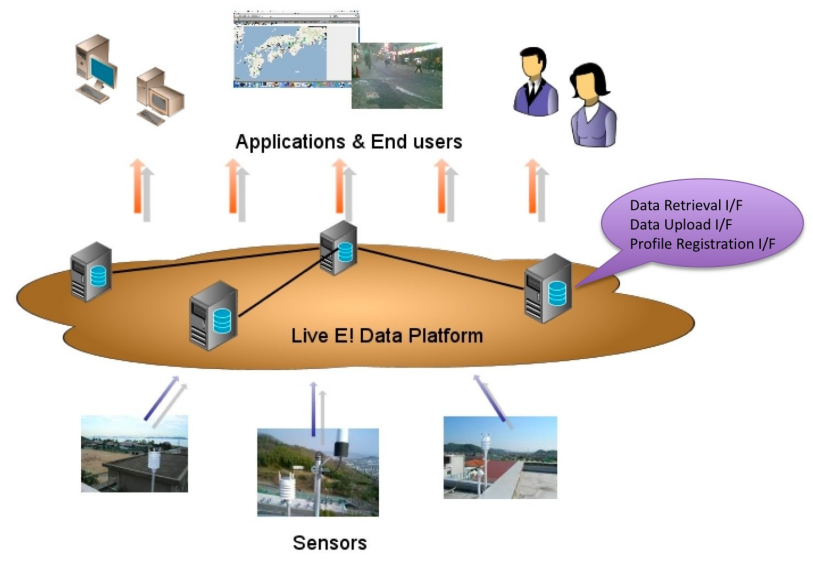

Figure 1: Live E! System Architecture

\section{RAINWATCH PROJECT}

RainWatch Project is one of Live E! Project applications. This project is aimed to transform raw collected data to ready-to-use information. It takes the Live E! Sensors' monitored rain states in user's location aware, analyzes relationship among known change-states at certain locations (e.g., rain to no_rain states) and finally reports warning or useful notification to the end-users which will certainly provide more benefits to the rain-sensitive end-users.

\section{A. System Overview}

The RainWatch system works as an application of Live E! Data platform. It periodically retrieves the current data from the platform, analyzes them and reports the result to the end-users. The end-user is bound to a location pointed by latitude and longitude coordinates, which in this paper referred as Point of Interest (POI). The POI entity in the RainWatch system registers all the sensors within its 20 kilometers radius and observes all the sensors' rain information. The POI summarizes the happening events in the area from the observed rain information and reports the following five statuses or events (like a watchdog) to the end-user:

(1) No rain in its proximity

(2) There is a rain in its proximity

(3) The rain is moving toward the POI

(4) The rain is moving outward from the POI

(5) It is raining in all area around POI.

In case a sensor in its range detects the rain, it reports "(2) There is a rain in its proximity". At the same time, the POI records the snapshot of rain-detected sensors and watches out for the next report. If there is more than one sensor detecting the rain, the POI will take (i) the location of detected sensors, (ii) the time of detection and (iii) the rain state into account for analyzing relationship among them. In the case that the distance of first detected sensor is greater than the second detected sensor, it reports, "(3) The rain is moving toward the POI". While the opposite case has occurred, it reports, "(4) The rain is moving outward form the POI".

\section{B. System Architecture and Implementation}

The RainWatch system architecture is illustrated in class diagram as in Figure 2. There are two main objects in the system; (1) Sensor node, (2) Point of Interest.

An area of interest covers the area of Kurashiki-city, approximately $25 \times 25$ square kilometers. A POI registers the sensor nodes which located in the area and classifies them according to its distance from the POI. The POI entity is also own two lists named as RainList and HistoryList. These RainList and HistoryList are the container of the same element type which is called RainListNode. The POI retrieves rain information from all registered sensors every 60 seconds. If it detects change in state at any sensor, it will create a RainListNode. A RainListNode keeps the following information: (1) sensors in which the state change has 
occurred, (2) the time of change, and (3) the status of change. For instance, in case of sensor detects change in state from no_rain state to rain state, the POI creates a RainListNode and adds it to both its RainList and its HistoryList. However, if the sensor detects change in state from rain state to no_rain state, the POI creates a RainListNode and adds it to the HistoryList, and removes the latest node with referred sensor from the RainList. Therefore, the RainList only keeps the information about currently raining nodes while the HistoryList records every change that occurred. This RainListNode is also used as input for the system to evaluate the relationship of detected cases and report for the users.

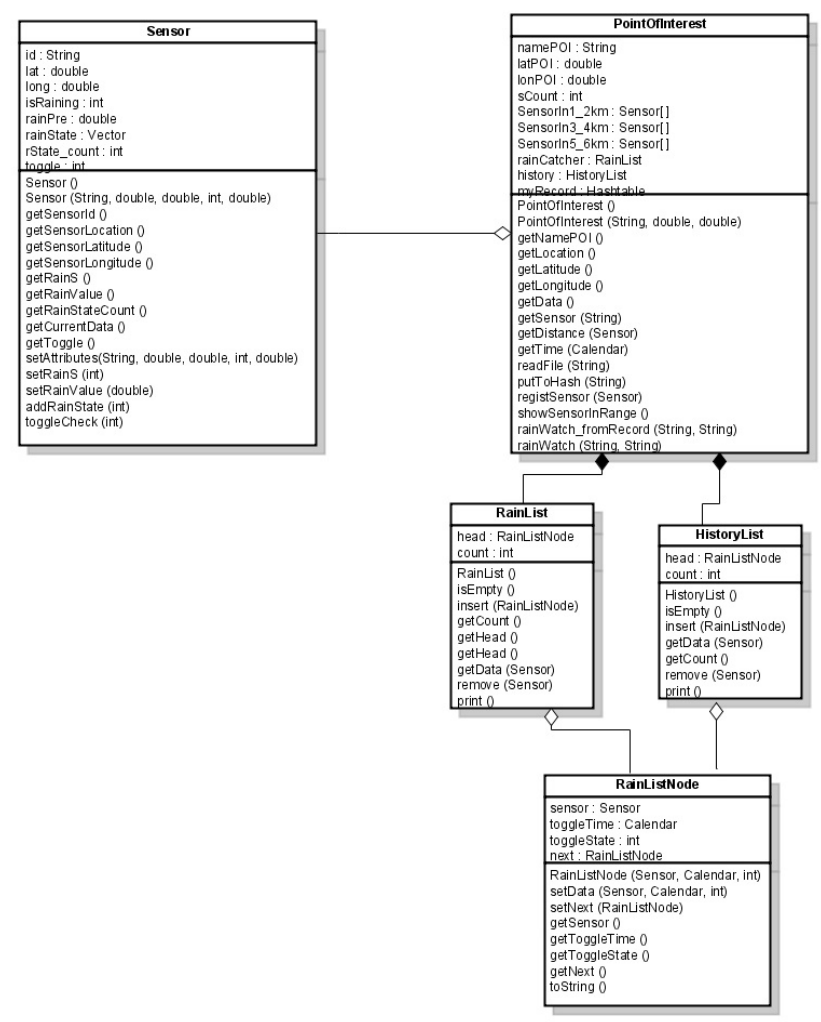

Figure 2: RainWatch Class Diagram

\section{Issues for the Internet-Based Sensor Networks}

Live E! Data platform, in the area we apply the RainWatch system, the data is updated every minute by means of Internet [4]. This feature allows the system to provide realtime detection and notification of rain to the endusers, which makes this system unique among other legacy rain forecast services. Thus, to make this system achieve their highest ability, the base-system should have efficiency in its real time data management for received location-aware queries. In addition, it should be able to manage large scale of the sensor system, which provides enormous dataset to the system.

\section{DISCUSSION}

RainWatch System has been tested with the pre-collected rain information data in Kurashiki-city which composed of 26 weather sensors from Live E! testbed. The duration of tested data ranges from 1 day (January 1, 2008) to 1 month (January 1, 2008 - February 2, 2008). On the test scenario, the system calls the pre-collected data in form of .csv file and puts them into the double hash tables according to timestamp and sensor-id. Then the system reads the data out according to timestamp, in the same way as working with realtime data. The system then analyses the data and output the result. The average performance is in $\mathrm{O}(\mathrm{n})$ yet the average memory usage will be vary according to the duration of tested data $\mathrm{O}\left(\mathrm{n}^{2}\right)$. However, on the completed system, it will work with the real-time data only, consequently there will be no need for extra storage beside for computation. From the experiment data, we have detected 13 rain events at Nishi Junior High School, which is referred, as our POI. 7 events are distinguished rain, and 6 are intermittent rain events with short discontinuity. The average time from first detected rain reports until the rain reaches POI is 16 minutes. Yet the prenotifications received at end-user range from 4 minutes to 34 minutes (Figure 3). The findings indicated that size of the observed area covered by sensors would have a direct impact on the length of pre-notification to the end-users. The larger coverage area with dense installations of sensors will definitely provide longer length of pre-notification which will allow users more time to get ready for the coming rain.

Time interval between the first rain detection to its arrival at POI

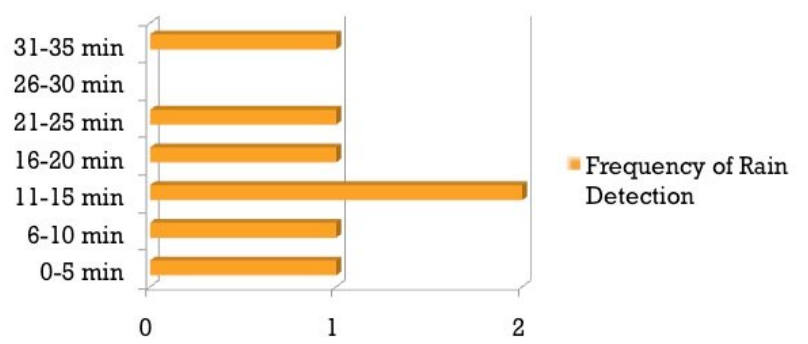

Figure 3: Time interval between the first rain detection to its arrival at POI (Tested from January 1, 2008 to February 2, 2008)

\section{CONCLUSION AND FUTURE WORKS}

RainWatch Project can help us to prepare for rain properly and prevent us from possible damage of lives and properties: e.g., the case of outdoor construction site, stores that sell products outdoor, umbrella seller, public sewer management [5]. In the current state, the RainWatch system only analyzes the rainfall status around POI, which might not be at best accuracy, but in the future, we are planning to enhance it to be finer and more accurate by taking other factors into account; e.g., wind speed, wind direction. We deploy the RainWatch system on the Live E! testbed around Kurashiki-city to evaluate its benefits as an application. If 
the result indicates its usefulness, we can further conclude that the base sensor network platform has a strong needs for supporting location-awareness in retrieving data as well as real-time updates with regard to scalability of the system, in other word, the number of deployed sensor nodes.

\section{REFERENCES}

[1] Automated Meteorological Data Acquisition System(AMeDAS),

Japan Meteorological Agency,

http://www.jma.go.jp/jma/en/Activities/forecast.html

[2] Live E! Project, http://www.live-e.org/

[3] S. Matsuura, H. Ishizuka, H. Ochiai, S. Doui, S. Ishida, M. Nakayama, H. Esaki and H. Sunahara, Live E! Project: establishment of infrastructure sharing environmental information, IEEE/IPSJ SAINT Workshop, 2007.

[4] ] H. Esaki and H. Sunahara, Live E! Project; Sensing the Earth with Internet Weather Station, IEEE/IPSJ SAINT, 2007.

[5] National Pollutant Discharge Elimination System (NPDES), http://cfpub.epa.gov/npdes/home.cfm?program id=5 